\title{
Metformin inhibits the growth of nasopharyngeal carcinoma cells and sensitizes the cells to radiation via inhibition of the DNA damage repair pathway
}

\author{
HAIWEN LI*, XINGGUI CHEN*, YING YU, ZHENNAN WANG, YUFANG ZUO, SHUHUI LI, \\ DONGHONG YANG, SHENGWEN HU, MEI XIANG, ZUMIN XU and ZHONGHUA YU
}

Cancer Center, Affiliated Hospital of Guangdong Medical College, Zhanjiang, Guangdong 524000, P.R. China

Received June 13, 2014; Accepted August 14, 2014

DOI: $10.3892 /$ or.2014.3485

\begin{abstract}
Nasopharyngeal carcinoma (NPC) is a leading cause of cancer-related mortality. Radiotherapy is one of the primary modalities for NPC treatment. However, in patients in the late stages of the disease, the local control rate and overall survival rate remain low. Therefore, it is urgent to identify new targets that can improve the outcome of radiotherapy in this neoplasm. In the present study, we investigated the effects of metformin on the radiosensitivity of NPC cells and explored the potential mechanisms. The radiosensitizing effects of metformin on NPC cells were measured by colony formation assay. Cell apoptosis was assessed by Hoechst 33342 staining analysis. DNA damage was detected by monitoring $\gamma$-H2AX foci with immunofluorescence. The changes in apotosisrelated and DNA damage repair-related proteins were detected by western blotting. Our study demonstrated that metformin significantly reduced the cell viability, enhanced radiosensitivity and potentiated radiation-induced caspase-9/-3 cleavage in the NPC cells. In addition, metformin plus radiation significantly upregulated the expression of p-ATM, p-ATR, $\gamma$-H2AX and downregulated the expression of ATM, ATR, p95/NBS1, Rad50, DNA-PK, Ku70 and Ku80. Therefore, our results suggest that metformin possesses a strong radiosensitizing potential in NPC cells. This radiosensitizing effect was associated with inhibition of DNA double-strand break repair processes through HR repair and the NHEJ repair signaling pathway, thereby enhancing radiation-induced cell apoptosis. These findings imply that metformin is a potent radiationsensitizing agent and may be a promising candidate for clinical
\end{abstract}

Correspondence to: Professor Zhonghua Yu or Dr Zumin Xu, Cancer Center, Affiliated Hospital of Guangdong Medical College, 57 South Renming Road, Zhanjiang, Guangdong 524000, P.R. China E-mail: zhonghua_yu@126.com

E-mail: zuminxu@gmail.com

*Contributed equally

Key words: nasopharyngeal carcinoma, metformin, DNA damage repair, radiosensitization, apoptosis evaluation as part of a combined regimen for the treatment of nasopharyngeal carcinoma.

\section{Introduction}

Nasopharyngeal carcinoma (NPC) is a leading cause of cancer-related mortality in Southeastern Asia, particularly in Southern China (1,2). Radiotherapy and concurrent chemoradiotherapy are standard modalities for NPC at the early stage and advanced stage, respectively (3). With the development of radiation techniques and chemotherapy modalities, the local control rate of patients in the early stage of the disease has risen to $70-90 \%$ over the past decade. However, in patients in the late stages of the disease (stages III-IV), the local control rate is only $50 \%$, with a 5 -year overall survival rate of $40-70 \%$ (4). The main causes of treatment failure are local recurrence and distant metastasis; the key factor is the presence of tumor cells possessing a resistance to radiation (5). Therefore, it is urgent to find novel, less toxic agents in combination with radiation to decrease radiation resistance and increase the radiation effect, as potential clinical candidates for this disease.

Metformin (1,1-dimethylbiguanide hydrochloride) is a widely used anti-diabetic drug (6). Recently, epidemiological analyses revealed that metformin reduced the cancer risk in diabetic patients (7-9). A number of preclinical studies have confirmed the anticancer activity of metformin with both in vitro and in vivo models (10). In addition, metformin has been reported to enhance the effects of chemotherapy $(11,12)$ and radiation (13-15). However, the effects of metformin combined with radiotherapy on NPC cells remain unknown.

In the present study, we investigated the actions of metformin on the radiosensitivity of NPC cells and explored the underlying mechanisms. Our results may contribute to the understanding of the mechanisms of action of the conventional drug and highlight potential implications of the combination of metformin with radiotherapy as an anticancer strategy.

\section{Materials and methods}

Cell culture and treatments. Three undifferentiated human NPC cell lines, CNE-2, HONE-1 and SUNE-1, were maintained by our laboratory and cultured in RPMI-1640 medium 
(HyClone, Logan, UT, USA) supplemented with $10 \%$ fetal bovine serum (FBS) (Biological Industries), $100 \mathrm{U} / \mathrm{ml}$ penicillin and $100 \mathrm{U} / \mathrm{ml}$ streptomycin in a humid atmosphere of $5 \% \mathrm{CO}_{2}$ at $37^{\circ} \mathrm{C}$.

Irradiation condition. The NPC cells were exposed to $4 \mathrm{MV}$ of X-rays with various doses of irradiation (0-8 Gy) using a linear accelerator (Eleketa, Stockholm, Sweden) with the source-skin-distance technique $(\mathrm{SSD}=100 \mathrm{~cm})$. The depth was set at $1 \mathrm{~cm}$ to the bottom of the 6-well plate or 6-cm dishes.

Cell proliferation assay. Cells in the early log phase were trypsinized and plated in a 96-well plate at a density of $1 \times 10^{4}$ cells per well. Twenty-four hours later, the medium was removed and replaced with fresh medium with metformin at the indicated concentrations $(0,4,8,16,25,32,50$ and $64 \mathrm{mM})$ for 24 or $48 \mathrm{~h}$ in the presence of $1 \%$ FBS. Cell density was measured using the CCK-8 (Dojindo Molecular Technologies, Japan) assay following the manufacturer's instructions. The absorbance of each well was determined at $450 \mathrm{~nm}$ using a microplate reader. The percentage of surviving cells from each group relative to the control were defined as the proliferation rate. For these studies, all experiments were repeated at least three times.

Colony formation assay. Cells in early log phase were trypsinized and plated in 6-well plates at 200, 400, 1,000, 2,000, and 4,000 cells per well and cultured overnight to allow for cell attachment. Then cells were treated with or without metformin for $24 \mathrm{~h}$ prior to administration of irradiation with the exposure dose corresponding to $0,2,4,6$, and 8 Gy. The cells were incubated for 10 days to allow for the formation of colonies. Cells were fixed and stained with $0.5 \%$ crystal violet (Sigma-Aldrich, St. Louis, MO, USA), and colonies containing $>50$ cells were counted. Survival curves were fitted using the multi-target click model in Graph Pad Prism 5.0 (GraphPad Software Inc., La Jolla, CA, USA). Each point on the survival curve represents the mean surviving fraction from at least three independent experiments.

Hoechst 33342 staining. Cells were grown on coverslips in 6 -well plates and treated with or without metformin for $24 \mathrm{~h}$ prior to administration of $6 \mathrm{~Gy}$ or sham radiation. The treated cells were cultured for another 12, 24 and $48 \mathrm{~h}$, fixed, and then stained with Hoechst 33342 (Beyotime Biotech, China) at a final concentration of $10 \mu \mathrm{g} / \mathrm{ml}$ for $15 \mathrm{~min}$, and scanned on a confocal microscope (magnification, x600 for the nuclear and morphologic analyses; Leica TCS SP5, Wetzlar, Germany). Apoptotic cells were identified by morphology and condensation and fragmentation of their nuclei. The percentage of apoptotic cells was calculated as the ratio of apoptotic cells to total cells counted, multiplied by 100 . Three independent experiments were conducted, and at least 300 cells were counted for each experiment.

Immunofluorescent staining. Cells $\left(2.5 \times 10^{5} / \mathrm{dish}\right)$ were plated onto sterile coverslips, and the following day were treated with or without metformin. Twelve hours later, cells were irradiated at a total dose of $6 \mathrm{~Gy}$. Cells were collected at the indicated time points (1,4 and $12 \mathrm{~h}$ ), washed, fixed and then blocked with
$5 \%$ BSA before incubation in rabbit monoclonal anti- $\gamma$-H2AX antibody overnight at $4^{\circ} \mathrm{C}$. After rinsing with PBS three times, the coverslips were incubated in secondary anti-rabbit Alexa Fluor 488 antibody (1:500; Invitrogen, Camarillo, CA, USA) for $1 \mathrm{~h}$ at room temperature. Then cells were stained with DAPI (Sigma-Aldrich) for $15 \mathrm{~min}$. Coverslips were then mounted onto slides with anti-fade mounting medium (Solarbio, China). The images were visualized, and representative views of the cells were recorded by a confocal microscope (Leica TCS SP5). For each treatment condition, the numbers of $\gamma-\mathrm{H} 2 \mathrm{AX}$ foci were counted for 50 cells at least. For the negative-control staining, the primary antibodies were omitted.

Western blot analysis. After treatment with metformin and/ or irradiation (6 Gy), sample preparation for immunoblotting was carried out as previously described (16). The membrane was then incubated with the appropriate primary antibody, anti-caspase-9, anti-caspase-3, anti-phospho-histone H2AX(Ser139), anti-ATM, anti-phospho-ATM(Ser1981), antiATR, anti-phospho-ATR(Ser428), anti-DNA-PK, anti-Ku70, anti-Ku80, anti-Rad50 and anti-p95/NBS1 (1:1,000; CST, Danvers, MA, USA), and anti- $\beta$-actin (1:1,500; Beyotime Biotech, China). The protein of interest was detected with goat anti-rabbit or anti-mouse IgG-horseradish peroxidase-conjugated secondary antibody (1:1,500; Beyotime Biotech, China). The band intensities were measured using Image J 1.41 software (NIH, Bethesda, MD, USA). Data are presented as the relative protein levels normalized to $\beta$-actin, and the ratio of the control samples was taken as 1.0 .

Statistical analysis. All data are expressed as mean values $\pm \mathrm{SD}$. For two-group comparison, the Student's t-test method was used. For more than a two-group comparison, one-way ANOVA was used. SPSS 13.0 software was used for all statistical analyses (SPSS, Chicago, IL, USA). P<0.05 was considered to indicate a statistically significant difference.

\section{Results}

Metformin inhibits the proliferation of NPC cells in a doseand time-dependent manner. CCK-8 assay was performed to evaluate the effects of metformin on the proliferation of NPC cell lines, CNE-2, HONE-1, and SUNE-1. Increasing concentrations of metformin and prolonged time from 24 to $48 \mathrm{~h}$ resulted in greater reduction in the cell viability of the three cell lines. It was revealed that metformin inhibited NPC cell proliferation in a dose- and time-dependent manner (Fig. 1A-C). The $50 \%$ inhibitory concentrations $\left(\mathrm{IC}_{50}\right)$ of metformin in the CNE-2, HONE-1 and SUNE-1 cells were $23.39 \pm 0.06,26.12 \pm 0.02$ and $24.75 \pm 0.04 \mathrm{mM}$, respectively. Metformin inhibited the proliferation of the three NPC cell lines slightly when at concentrations $<8 \mathrm{mM}$. Consequently, the metformin concentration of $5 \mathrm{mM}$ was thought to be a mild dose and was chosen to examine its radiosensitization effect in the following studies.

Metformin pretreatment followed by irradiation reduces the colony forming ability of NPC cells. A clonogenic assay was used to determine whether metformin enhances the radiosensitivity of NPC cells. Irradiation caused a dose-dependent 

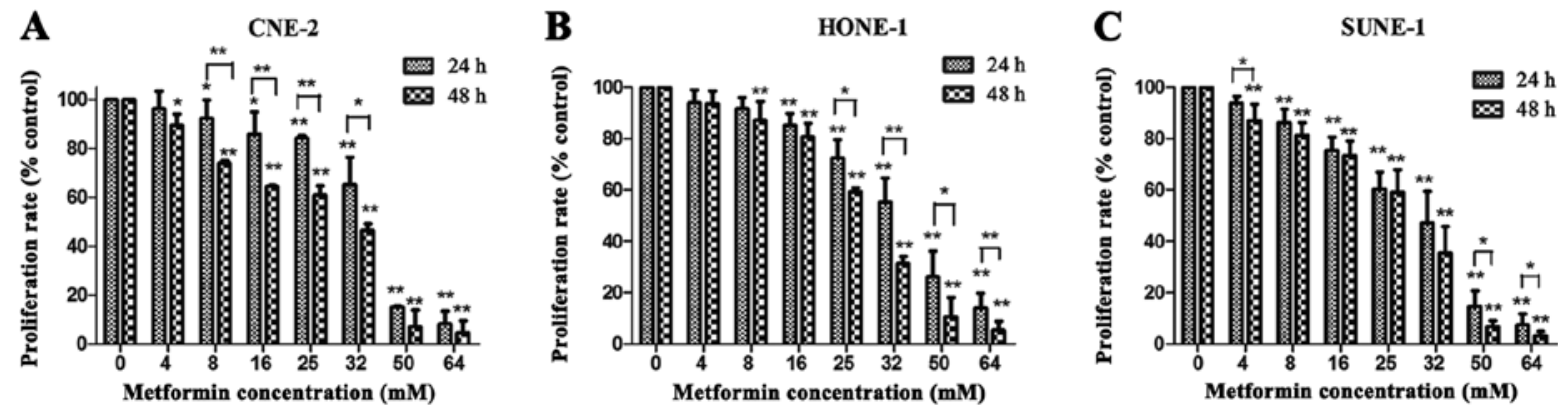

Figure 1. Effects of metformin on the proliferation of (A) CNE-2, (B) HONE-1 and (C) SUNE-1 NPC cells. NPC cells were treated with metformin at the indicated concentrations $(0,4,8,16,25,32,50$ and $64 \mathrm{mM})$ for 24 or $48 \mathrm{~h}$, and the proliferation rates were then determined by CCK- 8 assay. Metformin significantly reduced the viability of these cell lines in a dose- and time-dependent manner. All data are presented as the mean values \pm SD from three independent experiments. Cell proliferation in the untreated control cells was assigned as $100 \%$. ${ }^{*} \mathrm{P}<0.05 ;{ }^{* *} \mathrm{P}<0.01$ vs. control cells; bars, SD.

$\mathbf{A}$

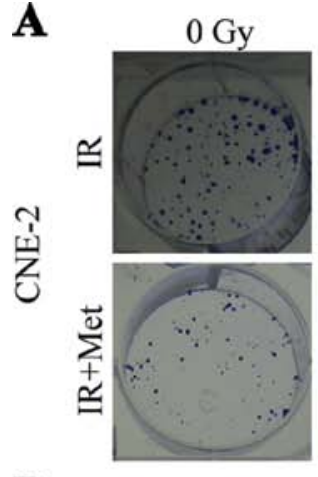

B

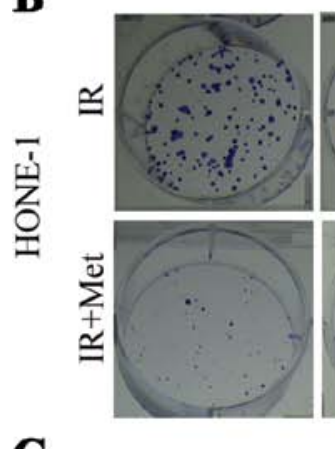

C

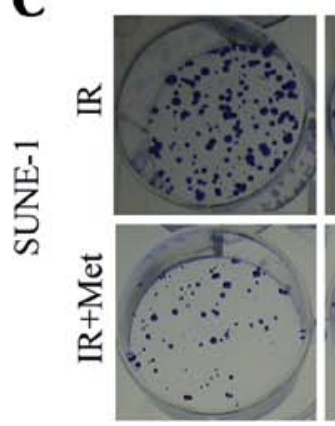

2 Gy
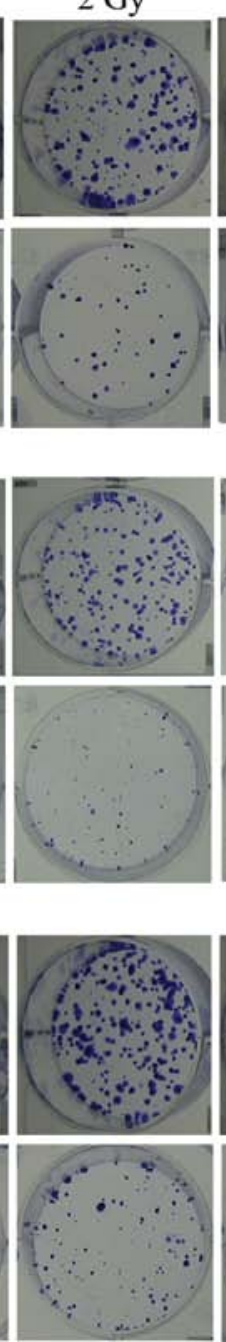
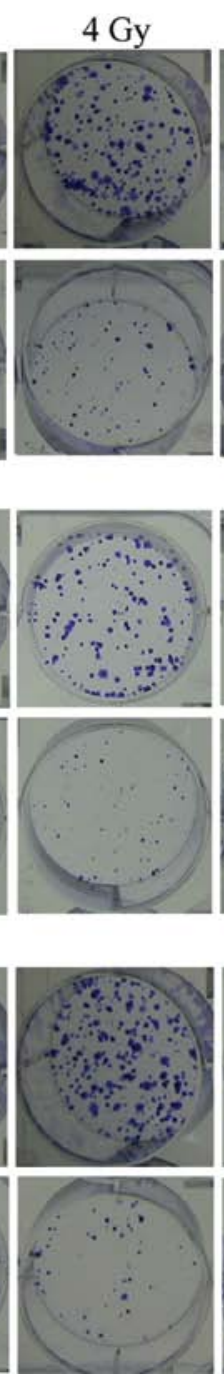
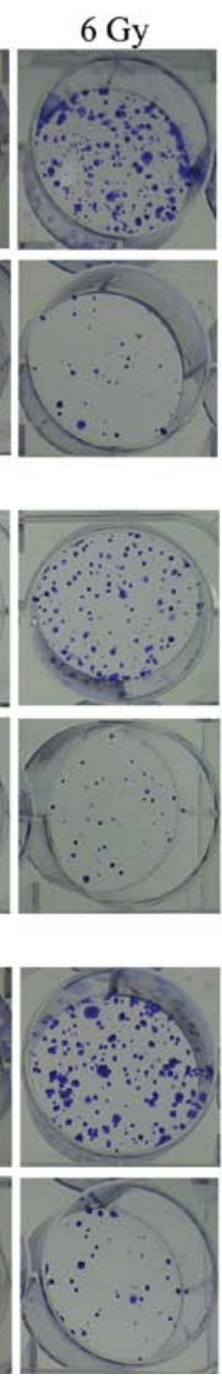

$8 \mathrm{~Gy}$
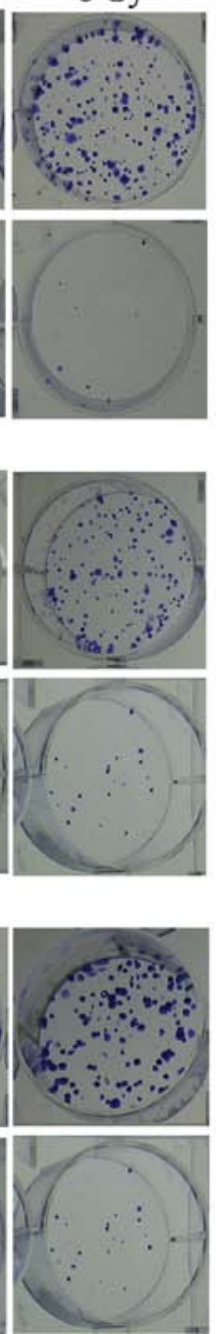

D

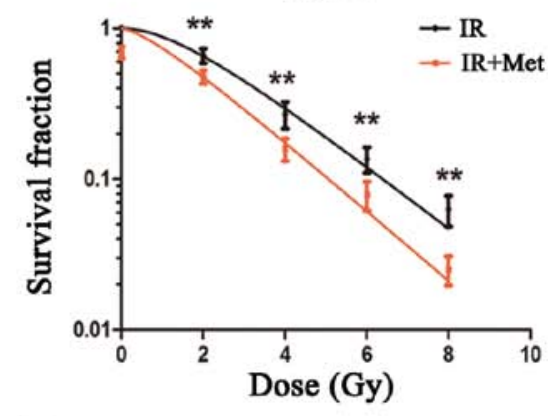

$\mathbf{E}$

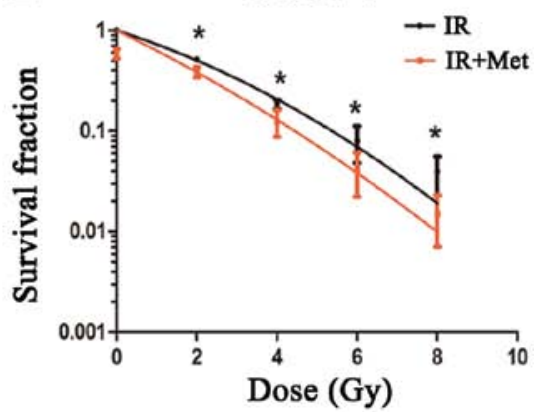

$\mathbf{F}$

SUNE-1

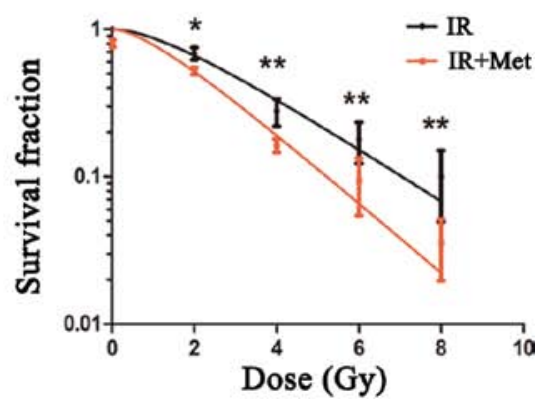

Figure 2. Effects of metformin pretreatment followed by irradiation on the survival fraction of NPC cells. Cells were treated with metformin or irradiation alone or pretreated with metformin for $24 \mathrm{~h}$ followed by 2 to $8 \mathrm{~Gy}$ irradiation and allowed to grow for 10 days. Representative images of colony formation of (A) CNE-2, (B) HONE-1 and (C) SUNE-1 cells are shown. Survival fractions of (D) CNE-2, (E) HONE-1 and (F) SUNE-1 cells were obtained from the results of the colony forming assays. Survival curves were fitted using the multi-target click model. Metformin markedly enhanced the radiosensitivity of these NPC cell lines with SER of 1.12 for CNE-2, 1.20 for HONE-1, and 1.22 for SUNE-1 cells, respectively. All data are presented as mean values \pm SD from three independent experiments. ${ }^{*} \mathrm{P}<0.05,{ }^{* *} \mathrm{P}<0.01$. IR, irradiation; Met, metformin; IR+Met, irradiation plus metformin.

reduction in clonogenic survival in the NPC cell lines, and we found significant variation in intrinsic radiosensitivity (Fig. 2). The radiation sensitivity was expressed as the surviving frac- tion at a clinically relevant dose of $2 \mathrm{~Gy}(\mathrm{SF} 2)$, and sensitization enhancement ratios (SER) were calculated. As shown in Fig. 2, the SF2 for the CNE-2 cells following IR and IR+Met was 

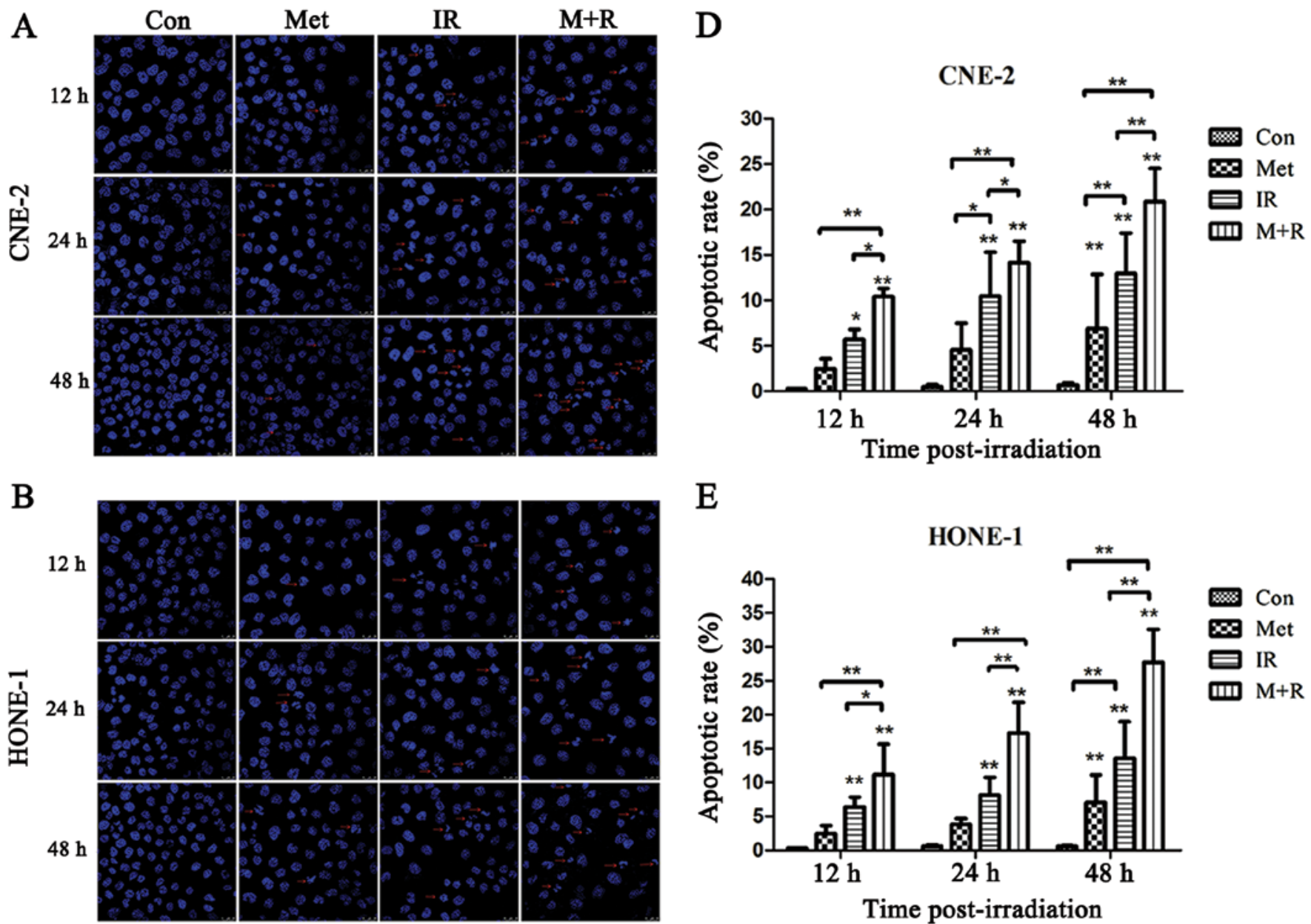

$\mathrm{E}$
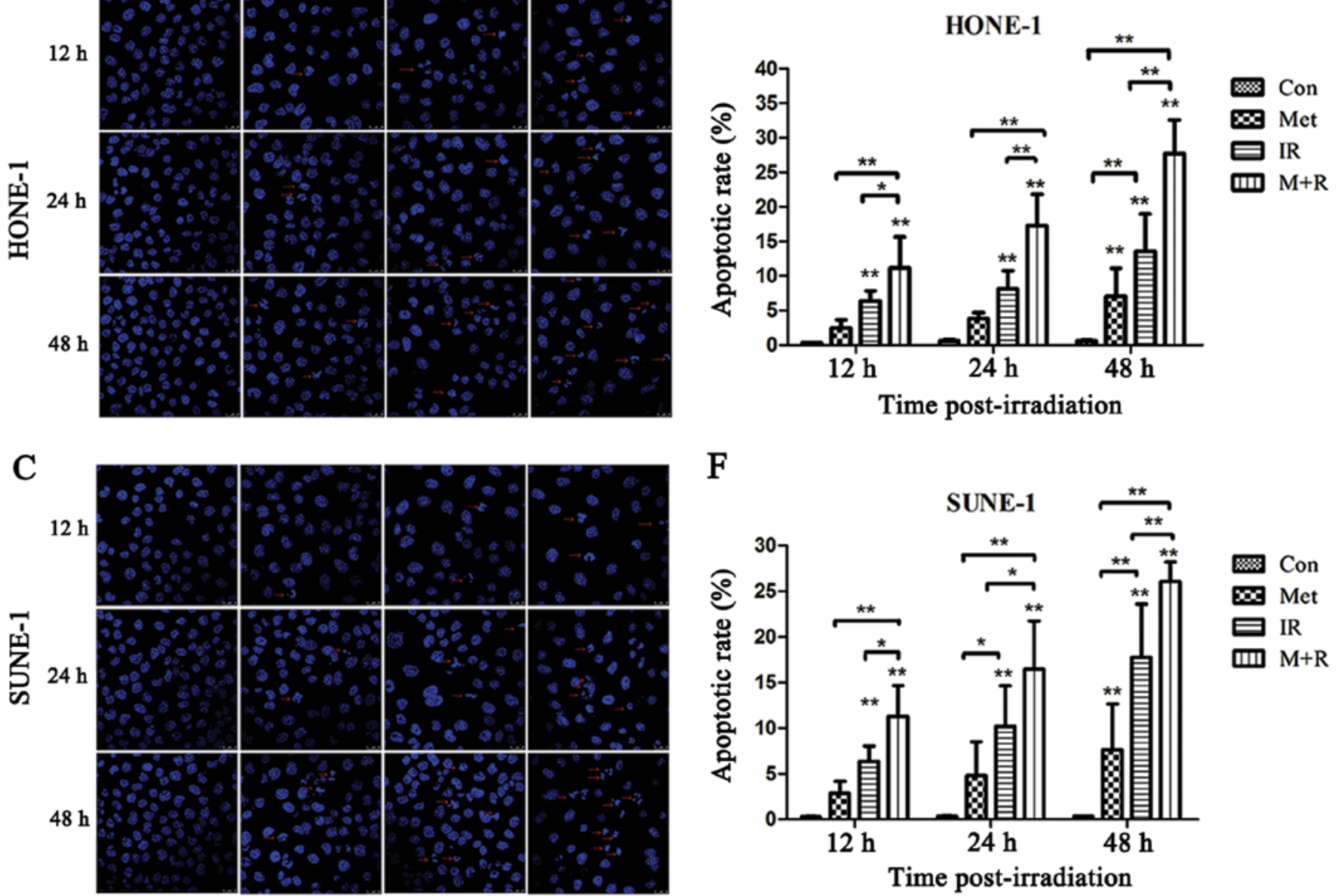

$\mathbf{F}$

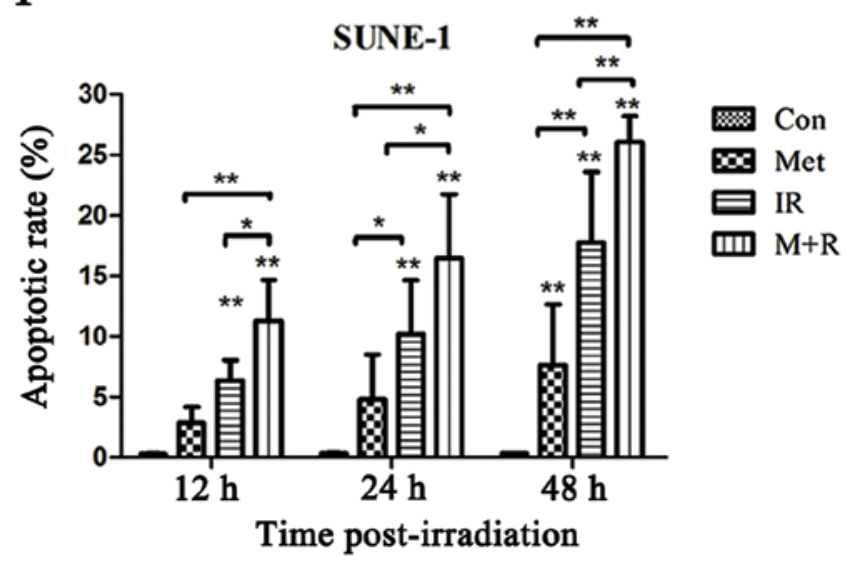

Figure 3. Effects of metformin combined with irradiation on the apoptosis of NPC cells. Cells were treated with metformin or irradiation alone or pretreated with metformin for $24 \mathrm{~h}$ followed by 6 Gy irradiation. Cells were fixed and nuclei were stained with Hoechst 33342 . Cell apoptosis was assayed by scoring cells presenting fragmented, damaged or condensed nuclei at 12, 24 and $48 \mathrm{~h}$ after the different treatments. (A-C) Representative images of apoptotic cells are shown. The red arrows indicate apoptotic cells. Magnification, x600. A total of 300 nuclei were analyzed in duplicate and each experiment was repeated at least three times. (D-F) The cell apoptotic rates were quantified. The apoptotic rate of the combined treatment increased significantly at 12,24 and $48 \mathrm{~h}$ and as time increased the apoptotic rate increased compared to the metformin or irradiation alone group. Data are shown as the mean values \pm SD from three independent experiments. ${ }^{*} \mathrm{P}<0.05 ;{ }^{* *} \mathrm{P}<0.01$.

$0.663 \pm 0.145$ and $0.478 \pm 0.097$, respectively $(\mathrm{P}=0.003)$. The $\mathrm{SF} 2$ for the HONE-1 cells following IR and IR+Met was $0.514 \pm 0.044$ and $0.387 \pm 0.079$, respectively $(\mathrm{P}=0.012)$. The SF2 for the SUNE-1 cells following IR and IR+Met was $0.686 \pm 0.132$ and $0.522 \pm 0.057$, respectively $(\mathrm{P}=0.012)$. When the dose increased the differences in the surviving fraction between the three cell populations became wider. Consistently, metformin markedly enhanced the radiosensitivity of NPC cell lines with SERs of 1.12 for CNE-2, 1.20 for HONE-1 and 1.22 for SUNE-1 cells.

Metformin pretreatment followed by irradiation increases cell apoptosis. To investigate whether the radiosensitization effect 

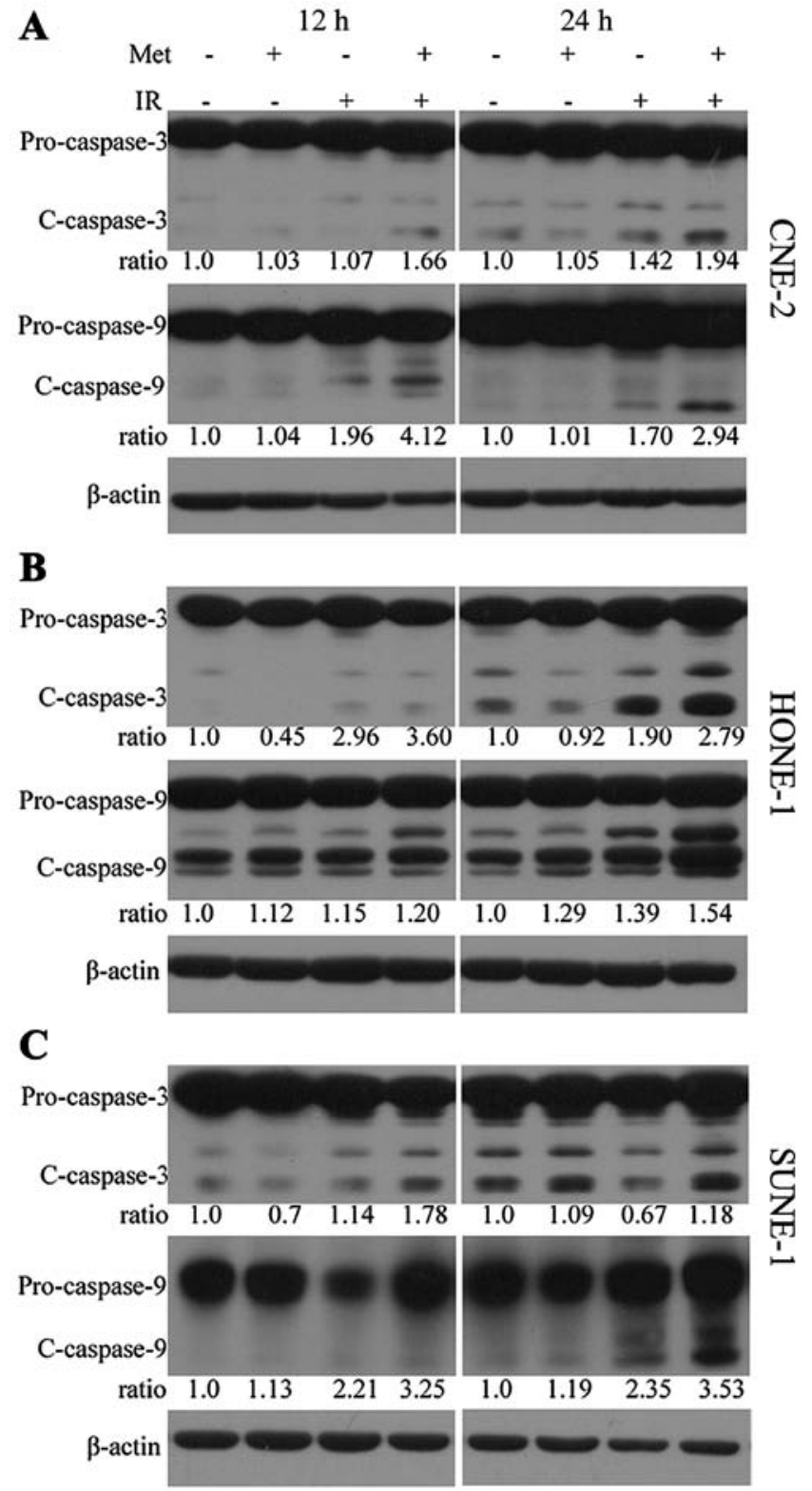

Figure 4. Effects of metformin combined with irradiation on the activation of caspase-9/-3 expression in NPC cells. Cells were treated with metformin or irradiation alone or pretreated with metformin for $24 \mathrm{~h}$ followed by $6 \mathrm{~Gy}$ irradiation. The levels of cleaved caspase-9/-3 were determined by western blotting at 12 and $24 \mathrm{~h}$ after irradiation. Protein levels were quantified using Image $\mathbf{J}$ software. Experiments were repeated at least 3 times and the representative results are shown. The cleavage of caspase- 3 and caspase- 9 proteins in cells following the combined treatments was markedly increased compared with that in the cells exposed to irradiation or metformin alone. The ratios below each blot are expressed as the proportion of cleavage band intensity to the total bands and calculated as follows: caspase- 9 or caspase- $3={ }^{*} \mathrm{Tc} / \mathrm{Tt}$, where $\mathrm{Tc}$ is the intensity value of the cleavage bands and $\mathrm{Tt}$ is the intensity value of the total bands.

of metformin is associated with cell apoptosis, the apoptosis of NPC cells was examined by examining morphological changes of nuclei by confocal microscopy at 12,24 and $48 \mathrm{~h}$ post-irradiation, and determining the levels of the caspase-3 and caspase- 9 by western blot assay. The apoptotic cells, which were characterized by condensed and fragmented nuclei, are shown in Fig. 3A-C. Further analysis revealed that metformin plus irradiation significantly increased cell apoptosis in all of the three NPC cell lines compared with cells exposed to irradiation alone over a certain time-period (Fig. 3D-F). The cleavage of caspase- 3 and caspase- 9 proteins were markedly increased in the cells treated with the combination of irradiation and metformin compared with the cells exposed to irradiation or metformin alone (Fig. 4A-C).

Metformin pretreatment followed by irradiation induces $\gamma-H 2 A X$ focus formation and increases the expression of $\gamma-H 2 A X . \gamma-\mathrm{H} 2 \mathrm{AX}$ has been identified as a marker of DNA double-strand breaks (DSBs). Immunocytochemical analysis using anti- $\gamma-\mathrm{H} 2 \mathrm{AX}$ antibodies was conducted in order to determine the effects of metformin on DNA repair. As shown in representative micrographs in Fig. 5A-C, the number of $\gamma$-H2AX foci was clearly distinguished after the different treatments. The mean number of $\gamma-\mathrm{H} 2 \mathrm{AX}$ foci per cell treated with irradiation or metformin alone was compared with the mean number of focu in cells treated with a combination of metformin and irradiation. The mean number of $\gamma-\mathrm{H} 2 \mathrm{AX}$ foci was shown to be increased in the cells treated with irradiation alone over time, while $\gamma$-H2AX foci in the cells exposed to metformin $(5 \mathrm{mM}, 12 \mathrm{~h})$ prior to irradiation were dramatically increased over a 1-, 4- and 12-h time course in the CNE-2 (Fig. 5D), HONE-1 (Fig. 5E), and SUNE-1 (Fig. 5F) cells. Consistently, metformin plus irradiation significantly increased the expression of $\gamma-\mathrm{H} 2 \mathrm{AX}$ protein compared with the expression in cells treated with metformin or irradiation alone (Fig. 6), as detected by western blotting.

Metformin pretreatment followed by irradiation affects the expression of DNA damage repair-associated proteins. Next, we investigated the effects of metformin on DNA damage repair proteins by western blotting. The protein levels of DNA damage repair-associated proteins in the CNE-2, HONE-1 and SUNE-1 cells under various conditions are shown in Fig. 7A. Clearly, in all cell lines, the expression levels of p-ATM(Ser1981) and p-ATR(Ser428) were upregulated, and the expression levels of ATM and ATR were downregulated, which indicated that DNA damage repair was inactivated over time. Furthermore, the expression level of DNA-PK was also significantly gradually downregulated over time (Fig. 7B). However, only a modest effect on the expression levels of Ku70, Ku80, Rad50, p95/NBS1 proteins was noted (Fig. 7). The combination of metformin with irradiation was far more effective than metformin or irradiation alone.

\section{Discussion}

In addition to the direct anticancer effects, metformin has also been reported to enhance the response of irradiation in several types of tumors, including fibrosarcoma (14), hepatoma (15), head and neck cancer (17), lung cancer and prostate cancer (13). Similarly, the present study reported for the first time that metformin markedly suppressed the proliferation of NPC cells (Fig. 1). Further investigation demonstrated that metformin combined with irradiation significantly decreased the clonogenic survival abilities and enhanced the radiosensitivity of NPC cells, with a sensitizing enhancement ratio (SER) of 1.12, 1.20 and 1.22 in CNE-2, HONE-1 and SUNE-1 cells, respectively (Fig. 2). Interestingly, metformin has also been evaluated in combination with radiotherapy or chemo- 
$\mathbf{A}$
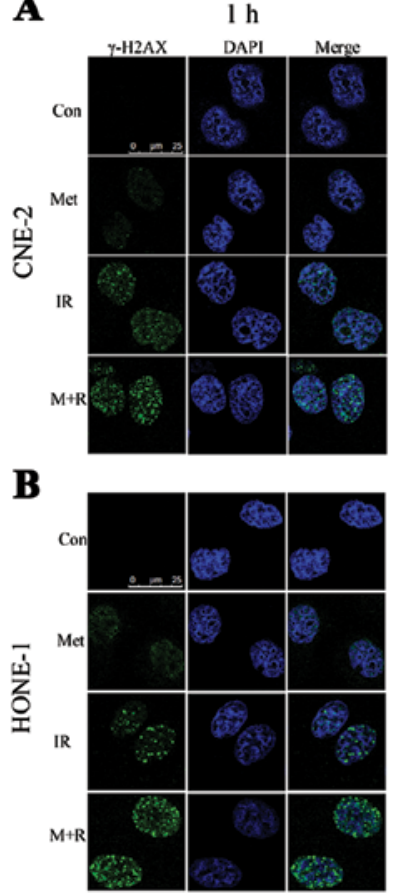

$\mathbf{C}$

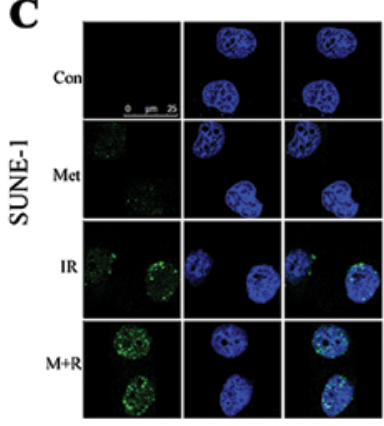

$4 \mathrm{~h}$
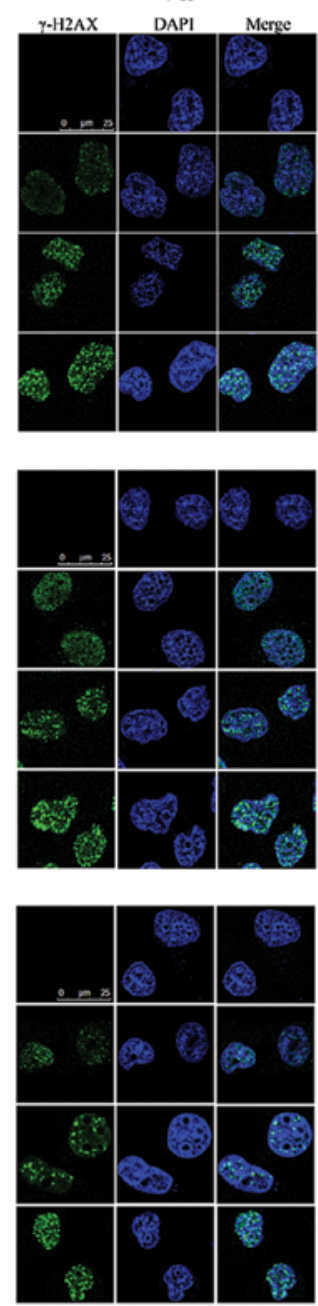

$12 \mathrm{~h}$
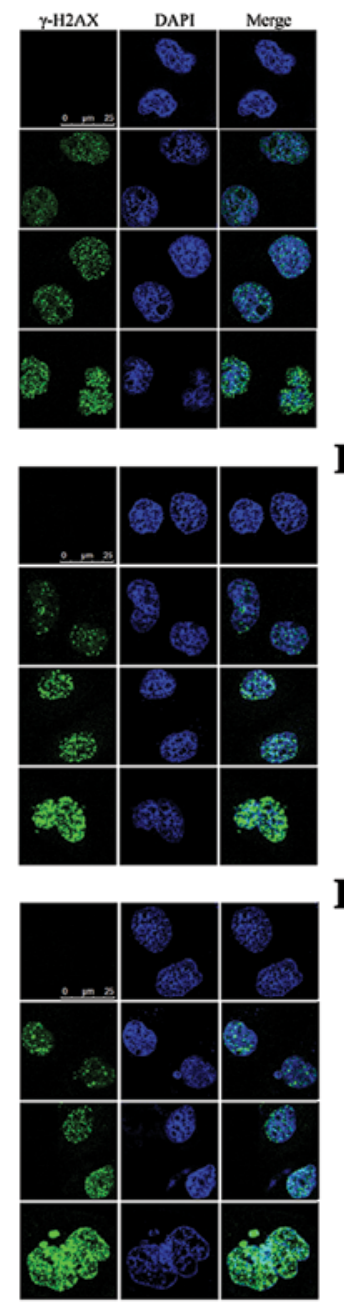

D

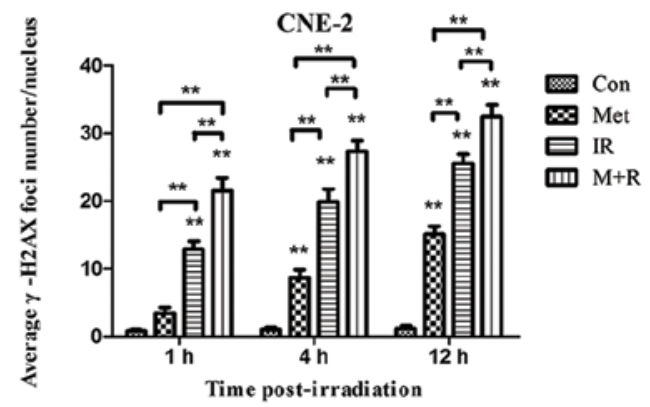

$\mathbf{E}$

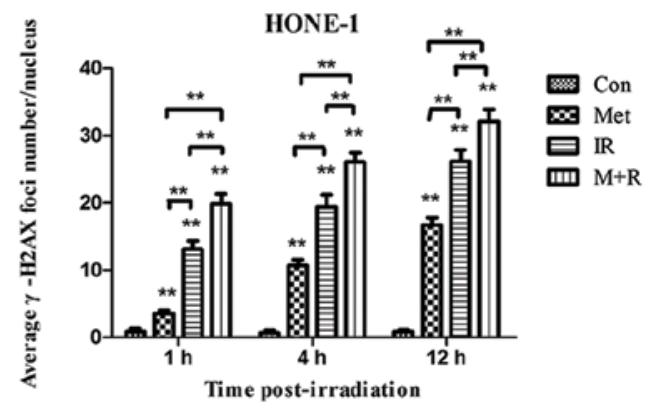

$\mathbf{F}$

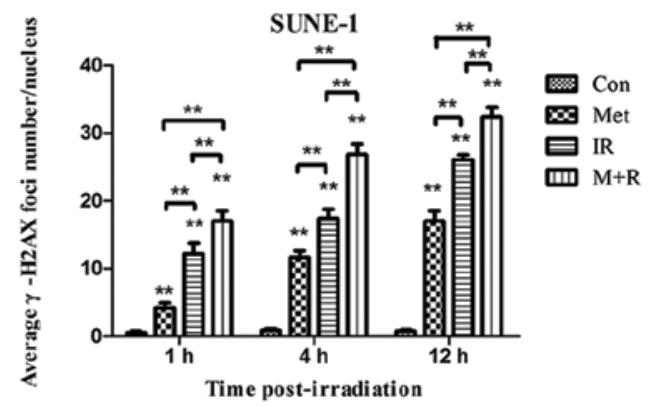

Figure 5. Effects of metformin pretreatment on irradiation-induced $\gamma$-H2AX focus formation in NPC cells. Cells growing on coverslips in 6-well plates were treated with metformin or irradiation alone or pretreated with metformin for $24 \mathrm{~h}$ followed by 6 -Gy irradiation and then stained for $\gamma$-H2AX foci. (A-C) Representative images of $\gamma$-H2AX foci in (A) CNE-2, (B) HONE-1, and (C) SUNE-1 cells. Scale bars represent $25 \mu$ m. (D-F) Quantification of the average number of $\gamma$-H2AX foci per nucleus at the indicated time points after various treatments. The mean number of $\gamma$-H2AX foci per cells treated with a combination of metformin and irradiation was significantly increased compared with irradiation or metformin alone in the different treatments followed by a 1- to 12-h post-irradiation duration. The average number of $\gamma$-H2AX foci per nucleus was determined randomly scoring 50 nuclei per sample, and each experiment was repeated at least three times. Merged foci were quantified and plotted for (D) CNE-2, (E) HONE-1 and (F) SUNE-1 cells at 1, 4, and 12 h post-irradiation. Columns, mean values $\pm \mathrm{SD}$ of three independent experiments. ${ }^{*} \mathrm{P}<0.05 ;{ }^{* *} \mathrm{P}<0.01$ vs. other groups at the same point of time.

radiotherapy in several clinical trials, and survival benefits were observed in metformin-treated patients compared to the non-metformin-treated patients. Skinner et al investigated the impacts of metformin on the outcome of radiation therapy for head and neck cancer. Their data showed that metformin use was significantly associated with decreased local recurrence rates (LRR) as well as improved overall survival (OS), with 5 -year OS rates of $87 \%$ and $41 \%$, respectively, for the patients receiving metformin and the remaining patients (17). This was further verified by another study, which found that metformin use was associated with a dose-dependent increased response to concurrent chemoradiotherapy in esophageal cancer with a higher pathologic complete response and a decrease in field locoregional failure (18). Given the above findings, the addition of metformin to radiotherapy might benefit the treatment of malignancies, including nasopharyngeal carcinoma.
Apoptosis has previously been regarded as a potential mechanism for radiosensitization. Lin et al showed that a molecularly targeted aurora kinase inhibitor, VE-465, significantly enhanced radiation-induced tumor growth suppression by a mechanism involving increased apoptosis (19). Grosse et al found that sunitinib combined with radiation induced apoptosis in follicular thyroid cancer cells via the intrinsic pathway of apoptosis (20). In addition, metformin has been reported to enhance irradiation-induced apoptosis and activate caspase-3 in lung cancer cells (21). In the present study, we additionally observed that metformin in combination with irradiation significantly increased the apoptotic rates (Fig. 3) and potentiated the cleavage of caspase-9/-3 proteins (Fig. 4) compared to either radiation or metformin treatment alone in NPC cells, which suggests that metformin may sensitize NPC cells to irradiation by promoting apoptosis. 


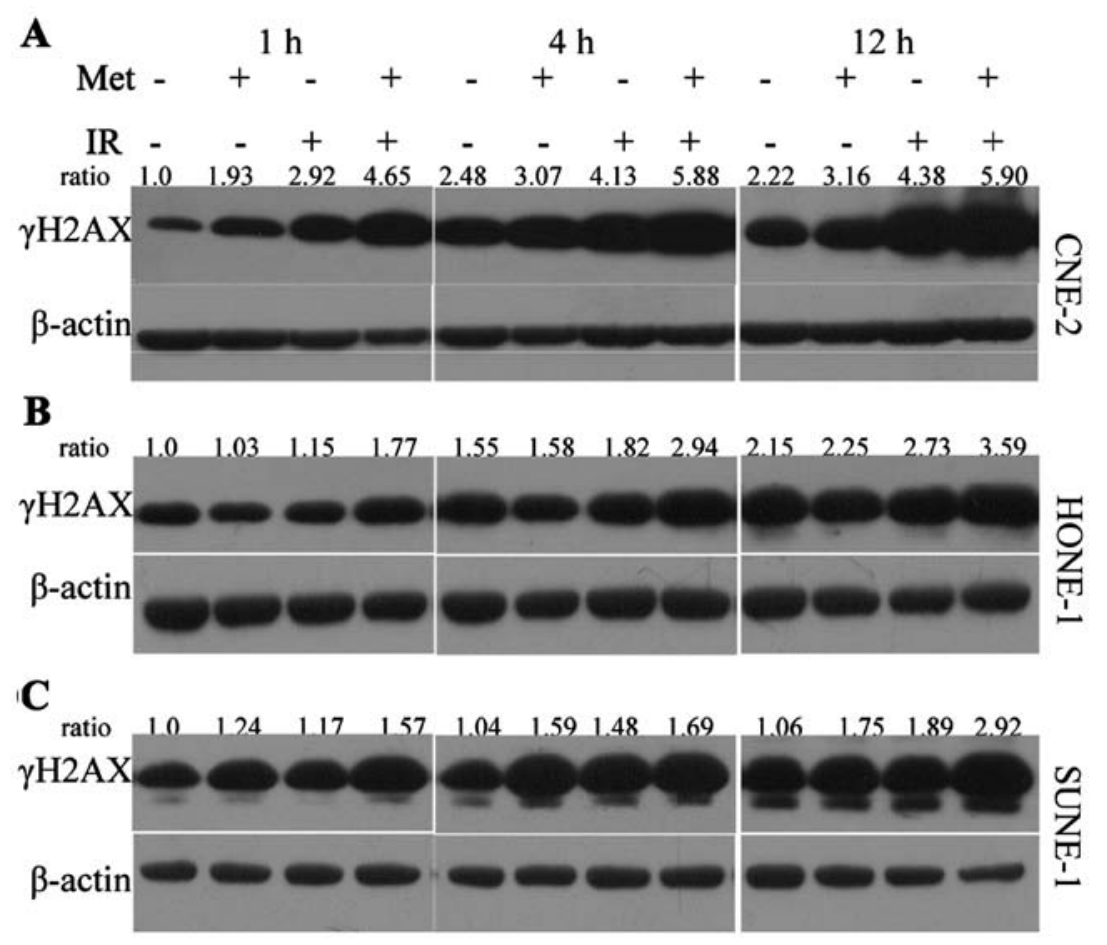

Figure 6. Effects of metformin combined with irradiation on the level of $\gamma$-H2AX expression in NPC cells. Cells were treated with metformin or irradiation alone or pretreated with metformin for $24 \mathrm{~h}$ followed by $6 \mathrm{~Gy}$ irradiation exposure. The levels of $\gamma$-H2AX protein were determined by western blotting at 1,4 and $12 \mathrm{~h}$ post-irradiation. Metformin plus irradiation significantly increased the expression of $\gamma-\mathrm{H} 2 \mathrm{AX}$ protein compared with metformin or irradiation alone. Protein levels were quantified using Image $\mathrm{J}$ software. Experiments were repeated at least 3 times, and the representative results are shown. The ratio above each blot is expressed as the intensity of the blot relative to that of the untreated control.

Radiotherapy is one of the major therapeutic strategies for cancer. Irradiation results in DNA damage and thus initiates a variety of signaling events in cancer cells (22). Double-strand breaks (DSBs) are one of the most important DNA damages caused by irradiation. In response to DSBs, histone H2AX is rapidly activated and phosphorylated. This phosphorylated form of $\mathrm{H} 2 \mathrm{AX}$ is named $\gamma-\mathrm{H} 2 \mathrm{AX}$. The expression of $\gamma-\mathrm{H} 2 \mathrm{AX}$ is a sensitive indicator of irradiation-induced DSBs, and $\gamma-\mathrm{H} 2 \mathrm{AX}$ foci are used to identify the number and location of DSBs and are widely used to evaluate cellular radiosensitivity $(23,24)$. To investigate whether metformin altered DSB repair, we monitored the formation of $\gamma-\mathrm{H} 2 \mathrm{AX}$ foci in cells treated with metformin or radiation. Our data indicated that the number of $\gamma-\mathrm{H} 2 \mathrm{AX}$ foci and the level of $\gamma-\mathrm{H} 2 \mathrm{AX}$ protein were significantly increased over time $(1,4,12 \mathrm{~h})$ following irradiation. Notably, although irradiation or metformin alone induced nuclear $\gamma-\mathrm{H} 2 \mathrm{AX}$ focus formation, metformin plus irradiation markedly induced an increased number of nuclear $\gamma$-H2AX foci compared to either irradiation or metformin treatment (Fig. 5A-F). Similarly, increased expression of $\gamma$-H2AX protein was observed in the NPC cells treated with metformin plus irradiation compared to either irradiation or metformin treatment alone as detected by immunoblotting (Fig. 6). Therefore, we concluded that metformin in combination with irradiation markedly induced DNA damage in NPC cells, suggesting that the radiosensitization effect of metformin might result from the augmentation of irradiationinduced DNA damage.

Recent studies have explored the potential mechanisms by which metformin enhances the response of irradiation in several tumors. It was reported that metformin sensitized cancer cells to irradiation by potentiating IR-induced AMPK activation $(13,14,21)$. Other studies found that following the combination of metformin and IR, enhancement of cytotoxic effects was noted by reducing ATP production (15), potentiating intracellular ROS levels induced by irradiation (25), and inducing senescence (17). To elucidate how metformin suppressed the repair of irradiation-induced DSBs, we investigated the effects of metformin on molecules involved in the non-homologous end-joining (NHEJ) and homologous recombination (HR) pathways by western blot assay. Our data demonstrated that metformin plus IR significantly induced time-dependent upregulation of p-ATM and downregulation of ATM thereby inhibiting DNA DSB repair pathways. These results implied that metformin induced radiosensitivity by decreasing the level of ATM expression. Furthermore, we examined a range of protein substrates, including ATR, NBS1, and Rad50 after DSBs were formed. Our findings showed that metformin pretreatment significantly reduced the level of ATR kinase, the phosphorylated form of ATR was strongly elevated, while the expression levels of Rad50 and NBS1 proteins were only modestly downregulated after exposure to irradiation (Fig. 7A). DNA-PK, Ku70 and Ku80 are critical proteins involved in non-homologous end-joining repair (NHEJ) of DNA DSBs. In the present study, we found that the expression levels of DNA-PK, Ku70 and Ku80 proteins were much lower following the combination treatment compared to metformin or irradiation alone, suggesting that metformin may sensitize radiation via interfering with the NHEJ pathway thus weakening DSB repair ability in NPC cells (Fig. 7B). Taken together, 
$\mathbf{A}$

CNE-2

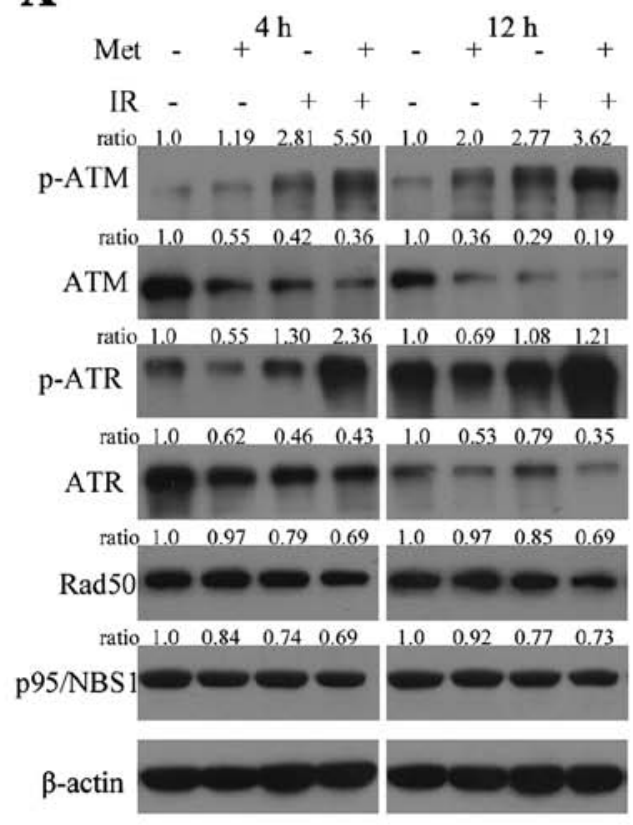

HONE-1

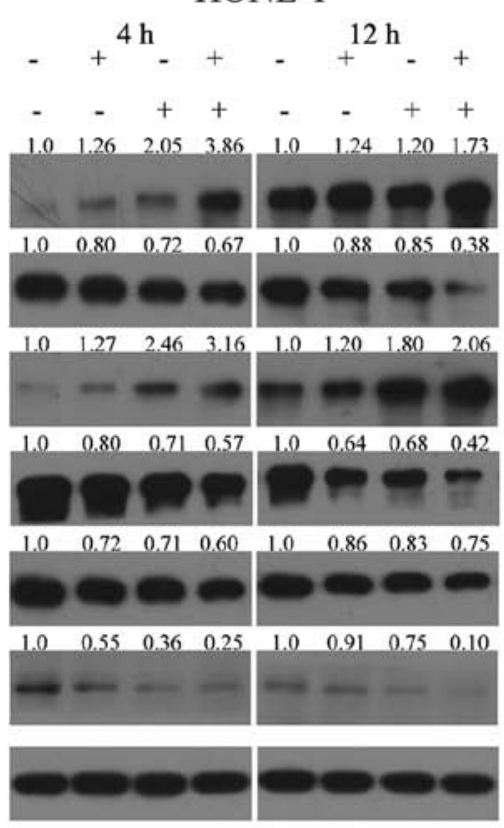

SUNE-1

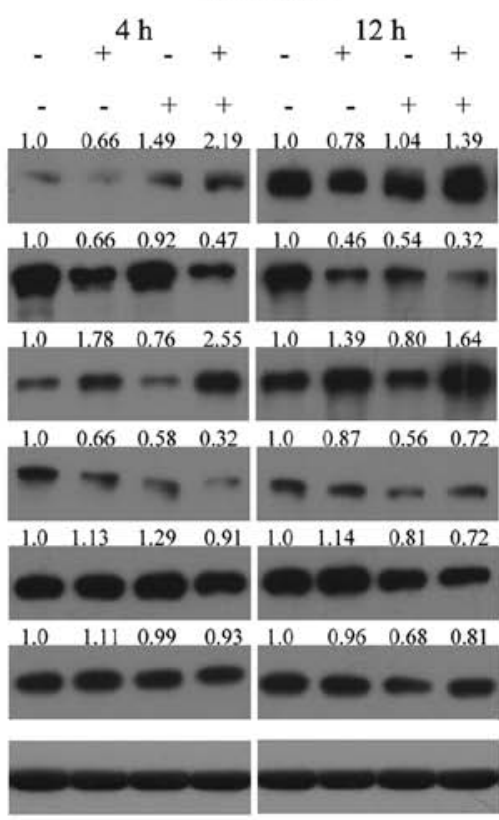

B

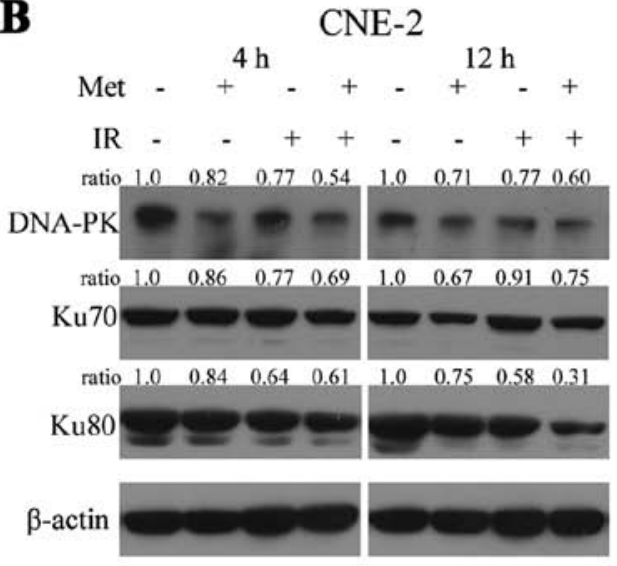

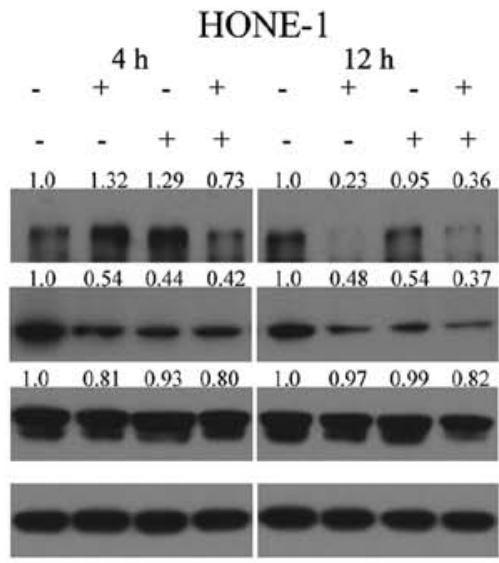

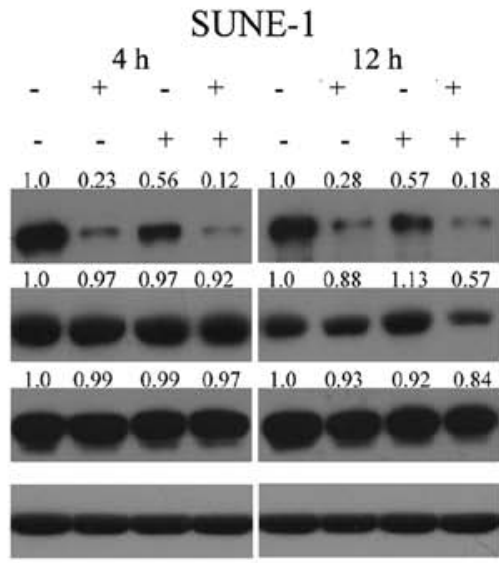

Figure 7. Effects of metformin combined with irradiation on the signals associated with the DNA damage repair pathway in NPC cells. Whole cell lysates were prepared and western blot analysis was performed using (A) anti-ATM, -p-ATM, -ATR, -p-ATR, -Rad50, and -p95/NBS1 antibodies for the HR pathway, and (B) anti-DNA-PK, -Ku70, and -Ku80 for the NHEJ pathway. In all cell lines, the expression levels of p-ATM(Ser1981) and p-ATR(Ser428) were upregulated, and the expression levels of ATM, ATR and DNA-PK were downregulated. However, only a modest effect on the expression levels of Ku70, Ku80, Rad50, p95/ NBS1 proteins over time was noted. The combination of metformin with irradiation was far more effective than metformin or irradiation alone. Experiments were repeated at least 3 times, and the representative results are shown. The ratios above each blot are expressed as the intensity of the blot relative to that of the untreated control.

these results indicate that metformin might be a dual inhibitor of both DNA-PKcs and ATM kinase, thus sensitizing NPC cells to irradiation by induction of more DNA damage and inhibition of repair of irradiation-induced DNA DSBs through diminishing NHEJ and HR pathways. For decades, researches have focused on the development of potent DNA-PKcs, and ATM inhibitors have yielded specific compounds, some of which have been found to be extremely useful in preclinical studies. Truman et al found downregulation of ATM protein resulting in an increase in radiation-induced apoptosis in human prostate cancer cells (26). Biddlestone-Thorpe et al demonstrated that ATM kinase inhibitor KU-60019 radiosensitized human glioma and mouse glioma stem cells via inhibition of the expression of ATM thereby inhibiting DNA repair capacity (27). In addition, Gil et al found that the dual
PI3K/mTOR inhibitor NVP-BEZ235 potently inhibited both DNA-PKcs and ATM kinases and attenuated the repair of IR-induced DNA damage in human glioblastoma, resulting in striking tumor radiosensitization (28). However, further exploration is required to clarify how metformin regulates ATM and DNA-PK.

In summary, this study demonstrated that metformin has a strong radiosensitizing potential in NPC cells. This radiosensitizing effect was associated with inhibition of DNA DSB repair processes through HR repair and NHEJ repair signaling pathways, thereby enhancing radiation-induced cell apoptosis. In addition, our data suggest that metformin is a potent radiation-sensitizing agent and may be a promising candidate for clinical evaluation as part of a combined regimen for the treatment of NPC. 


\section{Acknowledgements}

This study was supported by the National Natural Science Foundation of China (no. 81201736); the Science and Technology Planning Project of Guangdong Province, China (nos. KZ0710, 11401S009015, 11401S010013); and the Science and Technology Innovation Project of Guangdong Medical College, China (no. TD1124).

\section{References}

1. Yu MC and Yuan JM: Epidemiology of nasopharyngeal carcinoma. Semin Cancer Biol 12: 421-429, 2002.

2. Wei WI and Sham JS: Nasopharyngeal carcinoma. Lancet 365 2041-2054, 2005.

3. Chen L, Hu CS and Chen XZ, et al: Concurrent chemoradiotherapy plus adjuvant chemotherapy versus concurrent chemoradiotherapy alone in patients with locoregionally advanced nasopharyngeal carcinoma: a phase 3 multicentre randomised controlled trial. Lancet Oncol 13: 163-171, 2012.

4. Zhou J, Wang L, Xu X, Tu Y, Qin S and Yin Y: Antitumor activity of Endostar combined with radiation against human nasopharyngeal carcinoma in mouse xenograft models. Oncol Lett 4: 976-980, 2012.

5. Luftig M: Heavy LIFting: tumor promotion and radioresistance in NPC. J Clin Invest 123: 4999-5001, 2013.

6. Shaw RJ: Metformin trims fats to restore insulin sensitivity. Nat Med 19: 1570-1572, 2013.

7. Evans JM, Donnelly LA, Emslie-Smith AM, Alessi DR and Morris AD: Metformin and reduced risk of cancer in diabetic patients. BMJ 330: 1304-1305, 2005.

8. Landman GW, Kleefstra N, van Hateren KJ, Groenier KH, Gans RO and Bilo HJ: Metformin associated with lower cancer mortality in type 2 diabetes: ZODIAC-16. Diabetes Care 33 322-326, 2010

9. Singh S, Singh PP, Singh AG, Murad MH and Sanchez W: Antidiabetic medications and the risk of hepatocellular cancer: a systematic review and meta-analysis. Am J Gastroenterol 108 : 881-891, 2013.

10. Ben SI, Le Marchand-Brustel Y, Tanti JF and Bost F: Metformin in cancer therapy: a new perspective for an old antidiabetic drug? Mol Cancer Ther 9: 1092-1099, 2010.

11. Jiralerspong S, Palla SL and Giordano SH, et al: Metformin and pathologic complete responses to neoadjuvant chemotherapy in diabetic patients with breast cancer. J Clin Oncol 27: 3297-3302, 2009.

12. Iliopoulos D, Hirsch HA and Struhl K: Metformin decreases the dose of chemotherapy for prolonging tumor remission in mouse xenografts involving multiple cancer cell types. Cancer Res 71 : 3196-3201, 2011.

13. Sanli T, Rashid A and Liu C, et al: Ionizing radiation activates AMP-activated kinase (AMPK): a target for radiosensitization of human cancer cells. Int J Radiat Oncol Biol Phys 78: 221-229, 2010 .
14. Song CW, Lee H and Dings RP, et al: Metformin kills and radiosensitizes cancer cells and preferentially kills cancer stem cells. Sci Rep 2: 362, 2012.

15. Liu J, Hou M and Yuan T, et al: Enhanced cytotoxic effect of low doses of metformin combined with ionizing radiation on hepatoma cells via ATP deprivation and inhibition of DNA repair. Oncol Rep 28: 1406-1412, 2012.

16. Xu Z, Fang S, Zuo Y, et al: Combination of pigment epitheliumderived factor with radiotherapy enhances the antitumor effects on nasopharyngeal carcinoma by downregulating vascular endothelial growth factor expression and angiogenesis. Cancer Sci 102: 1789-1798, 2011.

17. Skinner HD, Sandulache VC, Ow TJ, et al: TP53 disruptive mutations lead to head and neck cancer treatment failure through inhibition of radiation-induced senescence. Clin Cancer Res 18: 290-300, 2012.

18. Skinner HD, McCurdy MR, Echeverria AE, et al: Metformin use and improved response to therapy in esophageal adenocarcinoma. Acta Oncol 52: 1002-1009, 2013.

19. Lin ZZ, Chou CH, Cheng AL, Liu WL and Chia-Hsien Cheng J: Radiosensitization by combining an aurora kinase inhibitor with radiotherapy in hepatocellular carcinoma through cell cycle interruption. Int J Cancer 135: 492-501, 2014.

20. Grosse J, Warnke E, Wehland M, et al: Mechanisms of apoptosis in irradiated and sunitinib-treated follicular thyroid cancer cells. Apoptosis 19: 480-490, 2014.

21. Storozhuk Y, Hopmans SN, Sanli T, et al: Metformin inhibits growth and enhances radiation response of non-small cell lung cancer (NSCLC) through ATM and AMPK. Br J Cancer 108: 2021-2032, 2013.

22. Jackson SP: Sensing and repairing DNA double-strand breaks. Carcinogenesis 23: 687-696, 2002.

23. Bonner WM, Redon CE, Dickey JS, et al: GammaH2AX and cancer. Nat Rev Cancer 8: 957-967, 2008.

24. Bourton EC, Plowman PN, Smith D, Arlett CF and Parris CN: Prolonged expression of the $\gamma-\mathrm{H} 2 \mathrm{AX}$ DNA repair biomarker correlates with excess acute and chronic toxicity from radiotherapy treatment. Int J Cancer 129: 2928-2934, 2011.

25. Sandulache VC, Skinner HD, Ow TJ, et al: Individualizing antimetabolic treatment strategies for head and neck squamous cell carcinoma based on TP53 mutational status. Cancer 118: 711-721, 2012.

26. Truman JP, Gueven N, Lavin M, et al: Down-regulation of ATM protein sensitizes human prostate cancer cells to radiation-induced apoptosis. J Biol Chem 280: 23262-23272, 2005.

27. Biddlestone-Thorpe L, Sajjad M, Rosenberg E, et al: ATM kinase inhibition preferentially sensitizes p53-mutant glioma to ionizing radiation. Clin Cancer Res 19: 3189-3200, 2013.

28. Gil del Alcazar CR, Hardebeck MC, Mukherjee B, et al: Inhibition of DNA double-strand break repair by the dual PI3K/ mTOR inhibitor NVP-BEZ235 as a strategy for radiosensitization of glioblastoma. Clin Cancer Res 20: 1235-1248, 2013. 